\title{
A stochastic movement simulator improves estimates of landscape connectivity
}

\author{
A. Coulon, ${ }^{1,2,8}$ J. Aben, ${ }^{3}$ S. C. F. Palmer, ${ }^{4}$ V. M. Stevens, ${ }^{5}$ T. Callens, ${ }^{6}$ D. Strubbe ${ }^{3}$ L. Lens, ${ }^{6}$ E. Matthysen, ${ }^{3}$ \\ M. Baguette, ${ }^{5,7}$ and J. M. J. Travis ${ }^{4}$ \\ ${ }^{1}$ Centre d'Ecologie et de Sciences de la Conservation (CESCO) UMR 7204, Bases Écologiques de la Conservation, \\ Muséum National d'Histoire Naturelle, 4 Avenue du Petit Château, 91800 Brunoy, France \\ ${ }^{2}$ Centre d'Ecologie Fonctionelle et Evolutive (CEFE) UMR 5175, CNRS-Université de Montpellier-Université Paul-Valéry \\ Montpellier-EPHE, Laboratoire Biogéographie et Ecologie des Vertébrés, CNRS, 1919 Route de Mende, 34293 Montpellier, France \\ ${ }^{3}$ University of Antwerp, Evolutionary Ecology Group, Groenenborgerlaan 171, 2020 Antwerp, Belgium \\ ${ }^{4}$ Institute of Biological and Environmental Sciences, University of Aberdeen, Zoology Building, Tillydrone Avenue, \\ Aberdeen AB24 $2 T Z$ United Kingdom \\ ${ }^{5}$ CNRS USR 2936. Station d'Ecologie Expérimentale du CNRS, 2 Route du CNRS, 09200 Moulis, France \\ ${ }^{6}$ Ghent University, Terrestrial Ecology Unit, Ledeganckstraat 35, B-9000 Gent, Belgium \\ ${ }^{7}$ Muséum National d'Histoire Naturelle, Institut de Systématique, Evolution et Biodiversité, UMR 7205, 57 Rue Cuvier, \\ F-75005 Paris cedex 5, France
}

\begin{abstract}
Conservation actions often focus on restoration or creation of natural areas designed to facilitate the movements of organisms among populations. To be efficient, these actions need to be based on reliable estimates or predictions of landscape connectivity. While circuit theory and least-cost paths (LCPs) are increasingly being used to estimate connectivity, these methods also have proven limitations. We compared their performance in predicting genetic connectivity with that of an alternative approach based on a simple, individual-based "stochastic movement simulator" (SMS). SMS predicts dispersal of organisms using the same landscape representation as LCPs and circuit theory-based estimates (i.e., a cost surface), while relaxing key LCP assumptions, namely individual omniscience of the landscape (by incorporating perceptual range) and the optimality of individual movements (by including stochasticity in simulated movements). The performance of the three estimators was assessed by the degree to which they correlated with genetic estimates of connectivity in two species with contrasting movement abilities (Cabanis's Greenbul, an Afrotropical forest bird species, and natterjack toad, an amphibian restricted to European sandy and heathland areas). For both species, the correlation between dispersal model and genetic data was substantially higher when SMS was used. Importantly, the results also demonstrate that the improvement gained by using SMS is robust both to variation in spatial resolution of the landscape and to uncertainty in the perceptual range model parameter. Integration of this individual-based approach with other developing methods in the field of connectivity research, such as graph theory, can yield rapid progress towards more robust connectivity indices and more effective recommendations for land management.
\end{abstract}

Key words: Cabanis's Greenbul; circuit theory; dispersal; Epidalea calamita; individual-based models; landscape genetics; least-cost paths; natterjack toad; Phyllastrephus cabanisi.

\section{INTRODUCTION}

The interplay between individual movement abilities (motivation, cognition, and motion capacities) and landscape properties (composition, configuration) determines to what extent habitat patches are functionally connected by animal dispersal (Taylor et al. 1993, Tischendorf and Fahrig 2000, Moilanen and Hanski 2001). Connectivity is a key determinant of movementbased processes and an important driver of spatial population dynamics (Wiens 2001), genetic structure

Manuscript received 3 September 2014; revised 18 December 2014; accepted 7 January 2015; final version received 9 February 2015. Corresponding Editor: H. H. Wagner.

${ }^{8}$ E-mail: acoulon@mnhn.fr and genetic diversity (e.g., Keyghobadi 2007), and ultimately species distributions. In the current context of rapid ongoing land use and climate change, connectivity has become an increasingly important factor in the persistence of species and the conservation of global biodiversity. First, habitat loss and fragmentation result in smaller, spatially segregated populations vulnerable to environmental, demographic, and genetic stochasticity, and inbreeding. Their probability of persistence is hence strongly dependent on sufficient inter-patch movements (e.g., Hanski and Gilpin 1991, Hanski 1998, Fahrig 2003). Second, in the face of climate change, the fate of species may often depend on their ability to track favorable environmental conditions in space and time (Hannah 2011, Hodgson et al. 2012, 
Travis et al. 2013), and habitat connectivity can play a key role in determining potential for range shifts (Opdam and Wascher 2004). Thus, understanding and mitigating the consequences of global changes on species persistence requires that we are able to estimate landscape connectivity accurately.

Connectivity estimators should reflect the actual relative numbers of movements among areas of interest (e.g., habitat patches) in a landscape, and, ideally, identify spatially the areas most frequently used for movements. To be reliable, they must represent not only the physical characteristics of landscapes (i.e., presence and configuration of the landscape elements), but also the effects of those characteristics on movement behavior of individuals (Taylor et al. 1993, Baguette et al. 2013). Currently, one of the most commonly used connectivity estimators is the least-cost path (LCP), which is based on a landscape grid where each landscape element is represented by a value reflecting the hypothesized cost of moving through it. The least costly route between predefined locations in the landscape (e.g., habitat patches or populations) is calculated, from which a measure (length or cumulative cost) of connectivity is derived (Adriaensen et al. 2003). The assignment of cost values to landscape elements apart, LCPs are easily calculated using a standard geographic information system or freely available statistical packages, which explains their popularity among researchers and land managers. However, the LCP approach implicitly assumes that individuals have perfect knowledge of the entire landscape (i.e., individual omniscience), allowing them to follow the optimal (i.e., least costly) route between any two locations. While LCPs may be better estimates of connectivity than simple structural connectivity estimates such as Euclidean distance, the additional variation explained is often low (e.g., Coulon et al. 2004, Stevens et al. 2006b).

Other connectivity metrics include those based on circuit theory, which make an analogy between individual movements in a spatial graph (where habitat patches are represented as nodes, and the movements among them as links) and the flux of electrons in an electric circuit (McRae and Beier 2007, McRae et al. 2008). This approach has the advantage of accounting for the presence of several possible pathways among patches (and not only one optimal path, as with LCPs). However, it is not based on behaviorally realistic movement rules. Realistic movement rules can be integrated through the use of individual-based models, which simulate multiple individual movements between patches within a landscape, and hence derive a connectivity estimate (Kool et al. 2013). A drawback of such models is that they usually require setting a large number of parameters, which impedes their use for many species for which detailed movement knowledge is lacking (Kool et al. 2013). One exception, though, is the "stochastic movement simulator" (SMS) (Palmer et al. 2011). This spatially explicit model simulates paths as a series of sequential movement decisions, which are functions of available landscape information within an individual's perceptual range. SMS uses the same landscape grid as LCP and circuit-based approaches, but, as movement paths are determined at the scale of a specified perceptual range (rather than at a landscape scale), they may not represent optimum routes between locations. SMS hence relaxes the assumption of individual omniscience, at the cost of two additional necessary parameters, one to control the degree of correlation in simulated movements (directional persistence), and one describing the distance at which animals can detect and respond to landscape properties (perceptual range). Additional parameters can (and sometimes may have to be added to) account for important biological characteristics of the species considered.

Here, we compared the performance of four connectivity estimates: (1) a simple measure of structural connectivity (Euclidean distances, i.e., the straight-line distances among locations), (2) the widely used LCP cumulative costs, (3) cumulative pairwise resistance distances (an estimate derived from circuit theory), and (4) pairwise dispersal rates simulated with SMS. The strength of the correlation between each and a genetic estimate of connectivity was taken as the indication of their relative performances. This comparison was conducted for two case study species in which dispersal had already been demonstrated to be affected by landscape structure (Stevens et al. 2006b, Aben et al. 2014). For both species, cost data of exceptional quality (based on objective assessments of the relative preference of the different types of landscape elements after experimental releases) and genetic data were already available. Moreover, the two study species, i.e., the Cabanis's Greenbul (Phyllastrephus cabanisi), a tropical forest bird, and the natterjack toad (Epidalea calamita), a temperate amphibian, present examples of contrasting movement abilities. Our analyses showed that SMS substantially outperforms the other three estimates, and that the improvement gained with SMS is robust to variation in spatial resolution and to uncertainty in the perceptual range.

\section{Methods}

\section{Study species and areas}

The Cabanis's Greenbul is a medium-sized, insectivorous passerine that inhabits central- to east-African moist forests (Keith et al. 1992). For this species, we quantified connectivity between four populations, each confined to cloud forest fragments located on a single mountain isolate of the Taita Hills in southeast Kenya (Appendix A). The landscape between forest fragments is a fine-grained mosaic of human settlements, terraced plots designed by smallholder farmers, tiny patches of indigenous forest, and exotic plantations (Pellikka et al. 2009). The species is relatively common within the four forest remnants, and capture-mark-recapture studies (since 1996) revealed that individuals can move between 
any of the remnant patches (across a maximum pairwise distance of $1170 \mathrm{~m}$ ). But at the same time, the Greenbul has been shown to be sensitive to the effects of forest fragmentation, exhibiting inhibition of movements through the matrix (Aben et al. 2012) and associated considerable genetic population differentiation among populations in the largest fragments (Callens et al. 2011).

The natterjack toad is a medium-sized terrestrial amphibian that breeds across western Europe in ephemeral ponds surrounded by open vegetation or bare ground (Beebee 1983). We used published information on gene flow among four toad populations inhabiting a Belgian agricultural landscape predominantly consisting of fields, pastures, and small villages interspersed with forest fragments (Appendix B) (Stevens et al. 2006b). In the study area, toad reproduction is mainly restricted to ephemeral ponds in artificial habitats such as former gravel pits, where small, mostly temporary ponds are used for breeding. Gene flow in this species is predominantly driven by dispersal of toadlets. The four populations are separated by $>2 \mathrm{~km}$, each population using one or several ponds. Gene flow analyses revealed that dispersal among the four populations is rare (a range of 0.03 to 0.88 immigrant toads per generation), asymmetrical, and affected by landscape structure (Stevens et al. 2006b).

\section{The cost surface}

Greenbul.-The cost surface was built upon land cover information presented in Aben et al. (2012), which was based on aerial photographs (at a resolution of 0.5 $\mathrm{m})$ and converted into a map classified into seven land cover types (Appendix A). This map was converted to a raster grid at a 5-m cell size, in which roads were omitted (by replacing this landscape feature with the bordering land cover type; roads were shown to be of minor importance in step selection of translocated birds [Aben et al. 2012]). We hypothesized that landscape effects on connectivity are driven by nonrandom selection of landscape elements in the matrix. Cost values reflecting those preferences were based upon empirically measured effects of each type of landscape element on movement behavior of the Greenbul: movements of translocated individuals were recorded and step selection functions were used to assess the effects of landscape elements on step selection (see Aben et al. 2012). Aben et al. (2012) assessed the relative effect on movement behavior of all types of landscape elements present in the study, except for the category "bush," because the Aben et al. (2012) model did not converge when all types of landscape elements were considered simultaneously. Here, to obtain an estimate of the relative effect of bush on bird movement decisions, we calculated coefficients for this element by running an alternative model where we added the category "bush" and removed "exotic plantation." The model coefficients obtained for each type of landscape element were subsequently used to calculate their preference values, whereby indigenous forest was assigned a value of 1 while the other types of landscape elements were assigned a preference value according to differences in their model coefficients relative to indigenous forest (Appendix A). Built-up areas were assigned a value 100 times that of field, as this landscape element was regarded to represent an absolute barrier to bird movement.

Toads.-The cost surface was derived from Stevens et al. (2006b) and based upon a fine-scale land cover map of the study area classified into eight land cover types (Appendix B). This map was converted to a raster grid at a 3-m cell size. As for Greenbuls, we hypothesized that landscape effects on connectivity are driven by nonrandom selection of landscape elements (i.e., preference) (Stevens et al. 2006a). Cost values reflecting those preferences were derived from experimental releases of toadlets: individuals were released in a Yshaped experimental arena in which the two branches of the $\mathrm{Y}$ mimicked two different types of landscape element. Preference values were determined as the relative permeability of boundaries between the different types of landscape elements $(=100-$ mean (over the different types of landscape elements) percentage of toadlets that enter a landscape element when starting in another one) (Stevens et al. 2006a). Built-up areas were considered absolute barriers, and rivers and ponds were assigned intermediate values (Stevens et al. 2006b).

\section{Euclidean distance, LCP, and Circuitscape estimates}

Euclidean distances were estimated as the shortest forest fragment-to-forest fragment distance for Greenbuls (considered more adequate than centroid-to-centroid distances because forest fragments greatly vary in size). They were estimated as the distances between the centroids of breeding sites for toads.

Least-cost paths (again, from forest edge to forest edge for Greenbuls, and between centroids of toad breeding sites) were calculated in ArcGIS 10.0 (Environmental Systems Research Institute, Redlands, California, USA), and the cumulative cost of each path (i.e., the "cost distance") was used as an estimate of pairwise patch connectivity.

Finally, connectivity estimates based on circuit theory were calculated with Circuitscape (McRae et al. 2013). Pairwise resistance distances between habitat patches were calculated using the pairwise mode, with each cell connected to its eight neighbors.

\section{SMS estimates}

SMS (Palmer et al. 2011) models the paths followed by virtual individuals depending on the distribution of cost values within an individual's perceptual range, how these costs are assessed (how they are averaged within the perceptual range, i.e., as an arithmetic or harmonic mean), and on the specified degree of directional persistence, i.e., the tendency to follow a correlated path (Zollner and Lima 1999). (Note that Palmer et al. 
[2011] referred to this SMS parameter as "directional bias," but "directional persistence" (DP) is a more accurate term, as it does not imply the influence of any fixed location or direction implied by "bias"). Illustrations of the effects of the different parameters on path shapes can be found in Palmer et al. (2011). For each simulation, the number of individuals arriving in each of the non-source patches was recorded and taken as a unidirectional pairwise estimate of between-patch connectivity (i.e., SMS connectivity estimates are asymmetrical: the connectivity from patch $\mathrm{A}$ to $\mathrm{B}$ is different from the connectivity from patch B to A). In this study, the same basic model was used for both species, but certain adjustments were made to account for speciesspecific biology, as explained below and summarized in Appendix C. The code of the SMS model used for Greenbuls and toads is provided in Supplements 1 and 2 , respectively.

Greenbuls.-To account for the fact that both theoretical studies (Zollner and Lima 1999, Bartoń et al. 2012) and empirical observations (Delgado et al. 2009a) have shown that dispersing individuals follow highly correlated paths at scales exceeding those of individual steps, the original SMS (as published in Palmer et al. [2011]) was extended with a "dispersal bias" parameter, which was used to control the degree of an individual's effective displacement relative to its natal patch. In addition, to prevent a sharp turn corresponding to a single cell completely changing an animal's direction, the current direction was determined over a number of previous steps (controlled by memory size) rather than just the single previous step. Thus, individuals having large memory size (such as birds) are more likely to resume their original movement direction after a temporary change in direction (Aben et al. 2014). Based on a congruence assessment of SMS simulations with actual movement paths of homing Greenbuls in the same study landscape (Aben et al. 2014), we selected the following combination of SMS parameters and settings: harmonic mean method, perceptual range $=25 \mathrm{~m}$, directional persistence $=2.0$, memory size $=2$, number of steps allowed $=2$ million. As dispersal bias was not used in Aben et al. (2014), we ran SMS simulations for dispersal bias $=1.02,1.04,1.06$, $1.08,1.1,1.14$, and 1.18. Virtual dispersers $(10000)$ were released from the centroid of each fragment (the source patches). Landscape boundaries were totally reflective.

Toads.-Initially, we fixed perceptual range at $30 \mathrm{~m}$ (i.e., 10 cells; based on field observations) and used the harmonic mean method for calculating the effective costs within the perceptual range. The directional persistence parameter was varied systematically between 2.0 and 16.0 in steps of 2.0. For each value of directional persistence, 10000 virtual dispersers were released from each of the four breeding populations and allowed to move until they recruited into a site (including the natal site), emigrated from the landscape (its boundaries were partially absorbing, depending on the strength of the DP : boun- dary absorbance increased with increasing DP) or exceeded the maximum number of 500000 permitted steps. To simulate the presumed attraction of dispersing toads to adult males calling from breeding ponds during the breeding season, we defined a $1-\mathrm{km}$ buffer around each breeding site (Appendix B), and assumed that juveniles could recruit into a site only during a temporal "recruitment window" of $\sim 5-6$ weeks (M. Baguette and V. M. Stevens, personal field observations). Specifically, we allowed each juvenile to move for 90000 steps with the window closed, and then opened the window for 10000 steps (to represent $10 \%$ of the year). If the individual was within a buffer zone when the window opened, or moved into one during an open window, then an additional behavior was applied to the movement algorithm by which the path was biased towards the centroid of the site within the buffer zone. This "goal bias" parameter worked in a similar way to the directional persistence parameter, but was given a strongly influential fixed value of 5.0, which made it highly probable that the individual would move in an almost straight path to the breeding site and recruit there. However, if the window closed before the site was reached, the goal bias was switched off and recruitment was not permitted until the next window opened after a further 90000 steps. Five recruitment cycles were applied, and hence the maximum number of permitted steps was 500000 (although in practice, most individuals either recruited during the first window or emigrated from the landscape).

In order to assess the effect of this assumption of auditory attraction to breeding sites during the recruitment window, we also ran SMS without the $1-\mathrm{km}$ buffer zones. In those simulations, no recruitment window was applied; any juvenile toad whose path entered a breeding site (except its natal site) at any time up to the maximum permitted, recruited to that site. Finally, we inferred the sensitivity of SMS estimates of functional connectivity (using the toad data) to (1) the spatial resolution at which the landscape was gridded and (2) the presumed size of perceptual range. For (1), we created additional rasters with cell sizes of $6 \mathrm{~m}, 10 \mathrm{~m}, 15 \mathrm{~m}$, and $30 \mathrm{~m}$, and ran SMS as above on these landscapes. Perceptual range remained fixed at $30 \mathrm{~m}$, directional persistence values were reduced as cell size was increased to allow for reduced autocorrelation at larger spatial resolution, and the lengths of the closed and open recruitment windows were reduced in proportion to the increasing length of a single movement step. LCP and Circuitscape connectivities were also recalculated for these landscapes. For (2), we ran SMS for the broadest set of perceptual ranges allowing reasonable computation times: 9, 18, 24, 30, 36, and $42 \mathrm{~m}$; directional persistence was fixed at 10.0 (the optimum value for a cell size of $3 \mathrm{~m}$ when perceptual range was $30 \mathrm{~m}$ ).

\section{Genetic estimates of connectivity}

Pairwise directional genetic estimates of connectivity were inferred through the estimation of the number of 
FIG. 1. Mantel correlation coefficients reflecting the predictive accuracy of Euclidean distance, the least-cost path (LCP), Circuitscape resistance distance, and the stochastic movement simulator (SMS) in describing migration rates among populations of Cabanis's Greenbul. Correlations were obtained for cost surfaces based on relative habitat preference, gridded at a spatial resolution of $5 \mathrm{~m}$. For SMS, correlations were obtained for different values of the dispersal bias parameter and assuming a perceptual range of $25 \mathrm{~m}$ and a directional persistence of 2.0 .

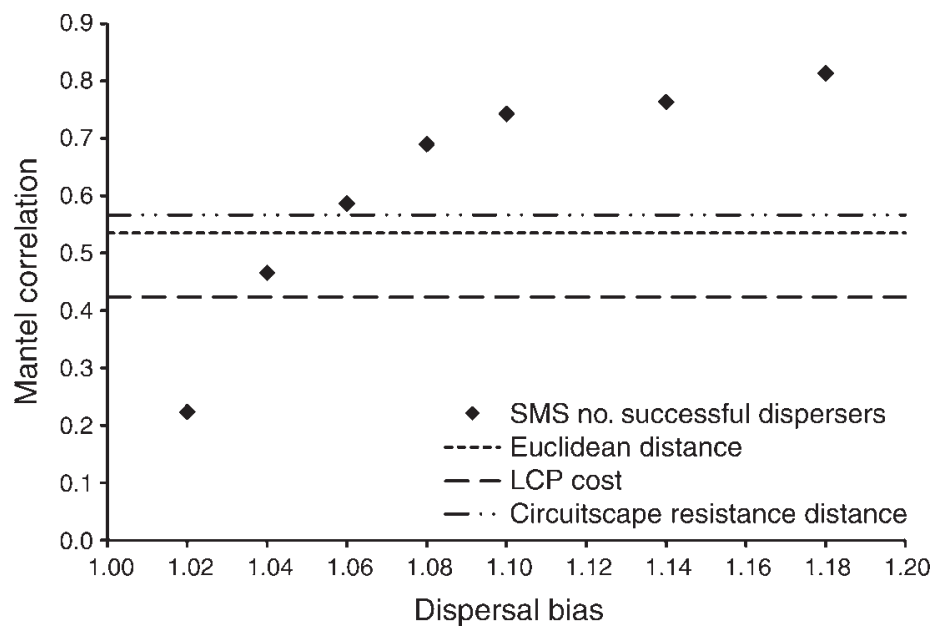

migrants per generation, as inferred from genetic samples.

Greenbuls.-We estimated migration rates based on variation at 10 microsatellite loci (see Callens et al. [2011] for details on the markers used). The genotyping procedure and the analyses performed to check genotype quality are described in Appendix D. We took advantage of our multi-year sampling scheme to use the program MLNE 2.3 (Wang and Whitlock 2003), which estimates migration between populations according to changes in allelic composition over a given time frame. In our case, two random subsets of genotypes were drawn from samples either taken between 1996 and 2000 ( $n=65$ for $\mathrm{CH}, 14$ for $\mathrm{FU}, 18$ for ND, and 65 for $\mathrm{NG}$; Appendix A) or between 2006 and $2010(n=55$ for $\mathrm{CH}$, 24 for FU, 16 for ND, and 54 for NG). Genetic samples were hence separated by a period of six years, which we assumed to correspond to one generation for Greenbuls. We ran open models in which we set each of the populations in the system as the source population, calculating pseudo-maximum-likelihood immigration rates $(m)$ and effective population sizes $\left(N_{\mathrm{e}}\right)$ for all focal populations, assuming a single generation interval between both time periods. Finally, the $N_{\mathrm{e}}$ estimates from the open models were multiplied by the obtained immigration rates $(m)$ to calculate the number of migrants per generation towards each population $(\mathrm{Nm})$.

Toads.-We used the estimated number of migrants per generation $(\mathrm{Nm})$ calculated in Stevens et al. (2006b). Those estimates were calculated with MIGRATE 0.7 (Beerli and Felsenstein 1999, 2001) from genetic samples from each of the four populations (28-43 individuals per site, genotyped at six microsatellite loci with 3-7 alleles per locus).

\section{Comparison of the estimate accuracy of the four connectivity models}

For both species, accuracy of the four connectivity models was evaluated based on Mantel correlations between the matrix of number of migrants per genera- tion $(\mathrm{Nm})$ and matrices of Euclidean distances, accumulated costs along the LCPs, Circuitscape resistance distances, and the number of virtual immigrants predicted by SMS for each combination of SMS parameters varied. When necessary, distance matrices were log-transformed to improve linearity for tests of statistical association. The Mantel correlations were calculated in SAS, v9.3 (SAS 2010) following Mantel (1967). The code of the SAS macro is provided in Supplement 3.

For toads, we also performed a visual comparison of summary maps produced by the four models, as described in Appendix E.

\section{RESUlts}

Greenbuls.-The connectivity estimates are provided in Appendix F. Genetic migration rates ranged from 3 to 31 individuals per generation. These rates were symmetric for the two largest fragments (1.04 ratio), whereas substantial asymmetry was found for the rates between population-pairs FU-CH (2.02 ratio), ND-NG (2.13 ratio) and FU-ND (5.00 ratio).

The correlation of functional connectivity estimated by SMS with the genetic estimates of connectivity depended on the dispersal bias: correlation was poor when dispersal bias was low (dispersal bias $=1.02, r=$ 0.224 ), but increased sharply with increasing dispersal bias until attaining an asymptote from dispersal bias $>1.10$ (maximum $r$ of 0.814 for dispersal bias 1.18) (Fig. 1). The correlation for LCP was even lower than that for Euclidean distance (Euclidean distance, $r=0.535$; LCP, $r=0.424)$, whereas that for Circuitscape resistance distance was slightly better than that for Euclidean distance (Circuitscape resistance distance, $r=0.567$ ). Correlations for SMS exceeded those for LCP cost, Euclidean distance, and Circuitscape resistance distance for dispersal bias values higher than 1.04 for the former and 1.06 for the latter two methods. At the optimum value of dispersal bias, SMS exceeded Euclidean 


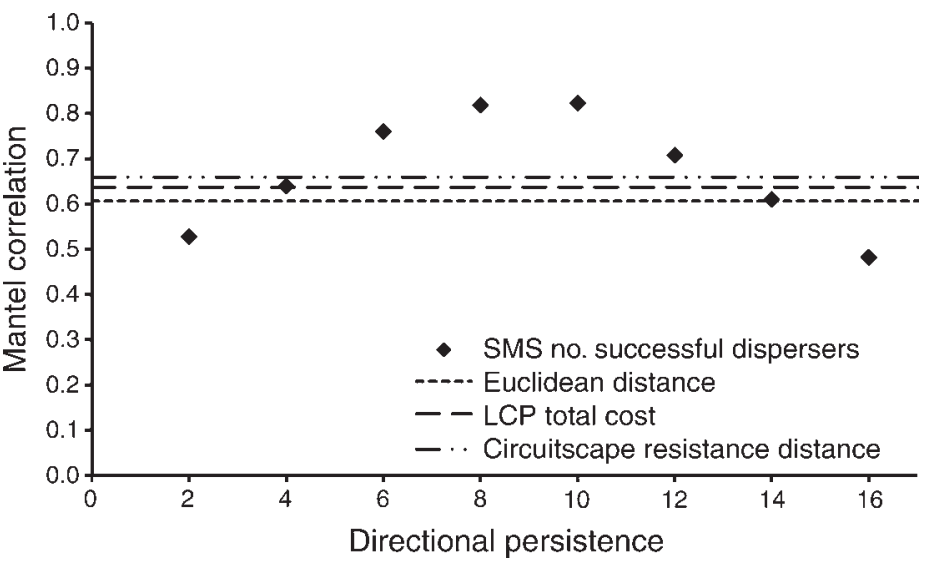

FIG. 2. Mantel correlations obtained by comparing the estimated number of migrants per generation of natterjack toads with connectivity estimates based on Euclidean distance, the least-cost path (LCP), Circuitscape resistance distance, and the stochastic movement simulator (SMS). Correlations were obtained for cost surfaces based on relative habitat preference, gridded at a spatial resolution of $3 \mathrm{~m}$. For SMS, correlations were obtained for different values of the directional persistence (DP) parameter and assuming a perceptual range of $30 \mathrm{~m}$. distance by $\Delta r=0.278$, LCP by $\Delta r=0.390$, and Circuitscape resistance distance by $\Delta r=0.247$.

Toads.-The connectivity estimates are provided in Appendix G. SMS estimates were highly sensitive to the value of the directional persistence parameter and correlated best with genetic migration rates at an intermediate value (directional persistence $=10.0$, maximum $r=0.823$ ) at which correlations exceeded those obtained for estimates of Euclidean distance, LCP cost, and Circuitscape resistance distance $(\Delta r=0.216, \Delta r$ $=0.186$, and $\Delta r=0.163$, respectively; Fig. 2). Removal of the effect of conspecific attraction on the movements of toadlets resulted in poor performance by SMS, worse than that of the other three methods (Fig. 3).

The correlation of functional connectivity estimated by SMS with genetic estimates was robust to the spatial resolution at which the cost landscape was represented (Fig. 4). Increasing cell size resulted in the SMS correlation peaking at lower values of directional persistence, but this did not change the general pattern of SMS outperforming the other connectivity models (maximum $\Delta r$ obtained for different resolutions ranged between $0.181-0.242,0.150-0.209$, and $0.126-0.187$ compared to Euclidean distance, LCP, and Circuitscape resistance distance, respectively). Compared to SMS, performance of LCP cost and Circuitscape resistance distance remained relatively constant across the range of resolutions tested (Fig. 4). Variation in perceptual range did not strongly alter SMS estimates, and performance remained well above Euclidean distance, LCP cost, and Circuitscape resistance distance across the range of perceptual range values examined (Appendix $\mathrm{H}$ ).

The visual comparison of summary maps produced by the four models showed that Circuitscape and SMS produce a more diffuse picture of connectivity between sites than straight-line Euclidean paths and LCPs. And contrary to Circuitscape, SMS predicts very poor connectivity between the NE sites and the SW sites. See Appendix E for maps and for a more detailed description and interpretation of those results.

\section{DiscusSION}

Connectivity is a rapidly growing research field of major importance for a range of ecological management decisions, including the design of ecological networks (e.g., Baguette et al. 2013), the control of invasive species (Glen et al. 2013), and prioritizing areas for restoration or protection from development (e.g., Donald and Evans 2006). Most frequently used methods for estimating connectivity rely either on Euclidean distance between patches of habitat, on estimates of LCP costs between those patches or on estimates of resistance

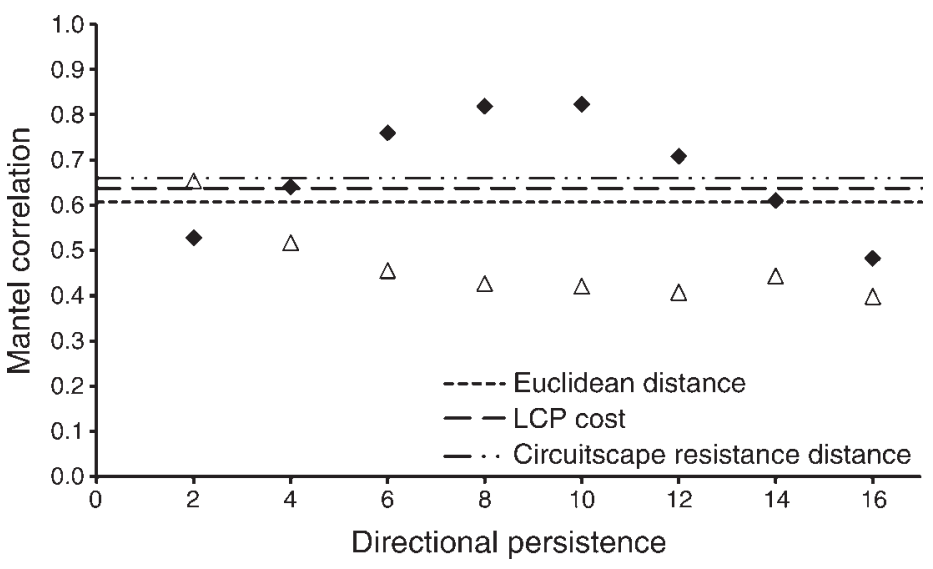

FIG. 3. Mantel correlations between the estimated number of migrants per generation of natterjack toads and predictions of the stochastic movement simulator (SMS) assuming a perceptual range of $30 \mathrm{~m}$ and using a spatial resolution of $3 \mathrm{~m}$ for the cost surface. Correlations reflect performance of SMS either assuming (diamonds) or not assuming (triangles) that dispersing toadlets are attracted to breeding sites by auditory conspecific attraction. For comparison, correlations based on Euclidean distance, leastcost path, and Circuitscape resistance distance estimates are also shown. 


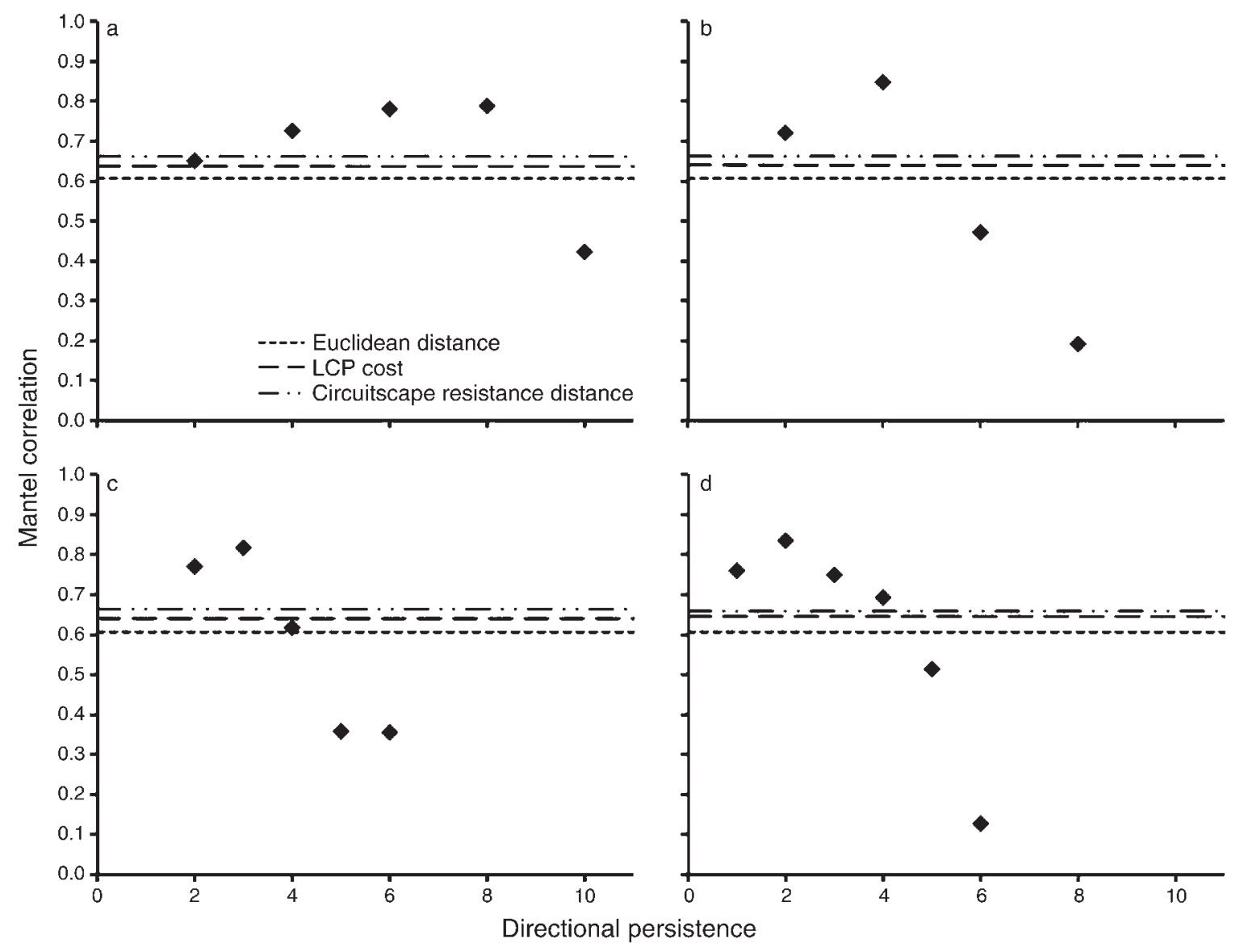

FIG. 4. Comparison of the Mantel correlation of the estimated number of migrants per generation of natterjack toads between sites with predictions of the stochastic movement simulator (SMS) (diamonds) assuming a perceptual range of $30 \mathrm{~m}$ and using cost landscapes gridded at four spatial resolutions: (a) $6 \mathrm{~m}$, (b) $10 \mathrm{~m}$, (c) $15 \mathrm{~m}$, (d) $30 \mathrm{~m}$ (compare with Fig. 2 for correlations at 3-m resolution). For comparison, correlations based on Euclidean distance, least-cost path (LCP), and Circuitscape resistance distance estimates are also shown (values for LCP at a 6-m spatial resolution: $0.637 ; 10 \mathrm{~m}$ : $0.639 ; 15 \mathrm{~m}$ : 0.640 ; $30 \mathrm{~m}$ : 0.645 ; values for Circuitscape resistance distance at a 6-m spatial resolution: 0.661; 10-m: 0.662; 15-m: 0.664; 30-m: 0.659).

between patches based on circuit theory. Here, we have illustrated that SMS can, by simple individual-based simulation of movement, provide a substantially better model of connectivity than either Euclidean, LCP, or circuit theory-based estimates. The rapid increase in availability of direct and indirect estimates of movement and dispersal behaviors (Tesson and Edelaar 2013) will facilitate the application of relatively simple stochastic movement models (such as SMS) in the future. Moreover, the integration of this approach with other developing methods in the field of connectivity research such as graph theory (McRae et al. 2008, Urban et al. 2009), can yield rapid progress towards more robust indices and more effective recommendations for management.

\section{Limitations of least-cost path estimates of connectivity}

There is a general recognition that estimates of connectivity that do not account for landscape characteristics between patches are likely to fail to represent well the relative connectivity between pairs of patches
(Kindlmann and Burel 2008). This has resulted in an increase in the number of studies making use of LCP estimates or extensions thereof. LCP-based methods typically provide a marginally improved model of interpatch movements (e.g., Coulon et al. 2004, Stevens et al. 2006b). However, as a model of inter-patch dispersal movements, LCP implicitly assumes that (1) individuals have complete knowledge of the landscape; (2) when they depart their natal patch, they know their desired destination; and (3) there is one optimal path, and the individuals use it (Palmer et al. 2011). One of the consequences of these assumptions is that LCP estimates of connectivity between two locations are symmetrical (i.e., patch-pairs share a single LCP) and that they are generally highly correlated with geographic distance (i.e., deviations from straight-line connections also come at a cost). Our results gained using SMS, a model developed specifically to relax these assumptions while retaining the landscape representation of LCP, emphasize the degree to which these embedded assumptions can limit the capacity of LCP-based approaches to 
predict inter-patch movements at scales relevant for animal dispersal (i.e., the landscape).

\section{Circuit-based estimates provide limited improvements}

As a consequence of the growing awareness of LCP's strong limitations, circuit-based estimates have been increasingly used in the past few years. Their main advantage over the LCP approach is their ability to account for the presence of multiple possible pathways among locations. Several studies have compared results from LCPs and circuit-based estimates, leading to contrasting results. It was suggested that circuit-based estimates perform better than LCPs, except in cases where populations are narrowly distributed along linear bands of suitable habitat (Schwartz et al. 2009, Moore et al. 2011). In our study circuit-based estimates performed better, although the improvement was relatively low. Circuit-based estimates do not integrate any behaviorally realistic movement rules, which may limit the accuracy with which they can model connectivity. This is confirmed by the fact that SMS best estimates substantially outperformed Circuitscape resistance distances. This result is a strong indication that the integration of realistic movement rules through individual-based models (IBMs) constitutes a necessary increase in complexity for obtaining more accurate connectivity estimates.

\section{"Simple" individual-based models: potential and caveats}

A potential constraint on the use of individual-based simulations of dispersal in connectivity research is the increased demands it makes in terms of data for parameterization (i.e., more parameters need to be set; e.g., Kool et al. [2013]). While complex simulations of individual behaviors are possible to parameterize for a small number of species for which large quantities of high-resolution data are available (e.g., Revilla et al. 2004), for individual-based models to be more widely applied they should ideally provide benefits over existing methods but impose limited additional data demands. SMS meets these requirements: it relaxes the unrealistic assumptions of LCP at the cost of only two additional necessary parameters (directional persistence and perceptual range) (one or two extra parameters, however, may have to be added to account for important biological characteristics of the species considered, as we discuss next).

Obviously, this simplicity limits the ability to simulate species-specific movement behavior explicitly, but our modeling of Greenbuls and toads demonstrates that even simple simulations can deliver substantially improved estimates of landscape connectivity. Importantly, the information needed on the landscape is exactly the same as is required for LCPs and circuit-based estimates, i.e., cost values. For the case studies presented here, these landscape cost values were deduced from experimental releases or analyses of relative preference of the landscape elements making up the study area
(Stevens et al. 2006b, Aben et al. 2012), and the cost surfaces used may therefore be regarded as reliable representations of the landscape (Zeller et al. 2012). Nevertheless, for both species we needed to add one additional piece of ecological knowledge for the model to perform well; this addition was the bias of movement away from the natal patch for Greenbuls (the dispersal bias parameter), and detectability of breeding sites beyond the specified perceptual range of toadlets in the breeding season. Accordingly, our expectation is that SMS will typically require some additional effort and dialogue between modelers and field ecologists with some biological knowledge of the species (or species group) in order for it to perform better than existing methods. This necessary integration between empirical studies and modeling is not unique to the SMS modeling approach and has been emphasized in other areas of research such as functional biodiversity (Jeltsch et al. 2013). The fact that SMS provides a worse fit than LCP and Circuitscape resistance distance in absence of these specific modifications, or when the directional persistence is too high or too low in the case of toads, provides a useful and general cautionary message for estimates of connectivity; we would not have identified this without testing our results against the genetic data. Given the relative ease with which genetic data can now be obtained, we believe that an iterative process of simulation, comparison with genetic data, and addition of extra simulated behavior, where necessary, ought comparatively easily to provide improved models for predicting the relative frequency of different inter-patch movements compared to those we currently use.

Ultimately, the process may be made more efficient through the use of formal inverse modeling techniques such as approximate Bayesian computation (Beaumont 2010), whereby the parameters such as directional persistence and perceptual range are estimated by fitting to the observed genetic data or to individual movements recorded by telemetry (e.g., GPS devices). Moreover, as more data are obtained from more taxa, and IBMs (e.g., SMS) are more and more used, the priors for their parameters will be progressively refined. This form of inverse modeling is one of the possible ways patterns can be used in the pattern-oriented modeling (POM) strategy (Grimm et al. 2005). The principle of the POM strategy in ecology is to use the large amounts of data contained in ecological patterns (such as time-series patterns or spatial patterns of presence/absence in patches), for example to optimize model structure through the inclusion in the model of the observed patterns that seem necessary to characterize the system, and of the variables and processes that are needed so that these patterns can emerge. Patterns can also be used to test and contrast theories, through the comparison of predicted and observed patterns for the different theories tested. Finally, patterns can help reduce parameter uncertainties, through inverse modeling, where calibration parameters are estimated by finding 
values that best reproduce observed patterns (Grimm et al. 2005). For example, Nabe-Nielsen et al. (2013) used a POM strategy to evaluate their model of harbor porpoise (Phocoena phocoena) foraging: they first parameterized a model of fine-scale movements and then tested whether the model was able to reproduce the large-scale movement patterns observed in nature. The POM strategy was advocated as having the potential to improve models substantially in applied ecology and conservation (Wiegand et al. 2003, Grimm et al. 2005).

\section{Insights about connectivity in Greenbul and natterjack toad populations}

The necessity to include species-specific refinements to the model is actually informative of the biology of the modeled system. In the case of the Greenbul, the addition of the dispersal bias parameter in SMS allowed us to vary the degree of directionality of Greenbul movements relative to their natal patch. Inter-patch movements and spatial gene flow can be a by-product of routine movements (e.g., to find food, shelter, etc.) or dispersal behavior per se (Van Dyck and Baguette 2005, Hovestadt et al. 2011). We anticipated that in the case of the Greenbul, which only very rarely ventures into the landscape matrix (Spanhove 2012), gene flow would be primarily driven by genuine dispersal movements. As Baguette and Van Dyck (2007) and Delgado et al. (2009b) showed, movements of dispersing individuals are generally more linear compared to routine movements. In our study we systematically increased the strength of dispersal bias from very weak (assumed to be more characteristic of routine movements) to relatively strong (assumed to be more characteristic of dispersal behavior). Our results clearly showed that predictions generated along that gradient differed substantially and that actual migration rates of this forest specialist bird species were far better explained when we assumed its movements to reflect dispersal behavior. It would be very interesting to see whether an optimum fit between estimates of gene flow and model predictions occurs at a weaker dispersal bias for forest generalist bird species which are more likely to use the matrix during daily routine movements.

In the case of the toad, the necessary implementation of an auditory attraction emphasizes the role of motivation in movement patterns and connectivity in general: depending on the specific aim of a movement, an individual may be more or less motivated to perform that move, and as a result use more direct itineraries, even though they go through areas of a priori higher resistance (with, for example, a higher danger level or with fewer resources [Bélisle 2005]). There has been very little empirical research on that area, but, for example, migrating female moose (Alces alces) move more quickly if they have calves, most likely to ensure arriving at safer areas and increase their calf survival (Singh and Ericsson 2014). Our results confirm that the presence, as informed by auditory cues, of potential breeding partners, increases landscape connectivity for the natterjack toad. It must be noted that the optimal value inferred for the directional persistence parameter cannot be interpreted biologically. Indeed, this value is intrinsically linked with the grain size used to represent the landscape: the smaller the grain size, the less likely individuals are to arrive into a patch if they have a low DP (because they will get lost in the matrix). This explains why the optimal DP decreases with increasing grain size (Fig. 4).

\section{Increased but reasonable computational demands}

A potential drawback of model complexity is the increased computational demands that are sometimes associated with it. In the toad case study, it is true that the computational times increased with model complexity. To get connectivity estimates for the two cost surfaces at a 3-m resolution on an Intel i5 Toshiba laptop, it took 5 minutes with LCPs, 70 minutes with Circuitscape, and 18 hours for SMS (testing nine levels of the DP parameter; see Methods: Euclidean distance, LCP, and Circuitscape estimates). However, the time to get SMS estimates was still not prohibitively long. As a result, we believe the balance between the accuracy of connectivity estimates and computation time is strongly in favor of SMS.

\section{Complementarity of SMS with graph-and circuit- based approaches}

The improved estimates of the connectivity between different pairs of habitat patches that SMS provides can be integrated within other landscape connectivity methods, including those based on graph theory. These methods require estimates for the strength of links between all pairs of habitat patches on the landscape, and SMS essentially provides an alternative method of calculating the strength of these linkages between a network's nodes. One can even imagine integrating SMS results with circuit-based estimates, replacing the "cost" raster with a raster inversely proportional to the number of SMS-simulated moves in each cell. Thus, these methods are strongly complementary; SMS (or any other simple movement simulator) provides a means for incorporating the increasing knowledge and information available on dispersal behaviors, while the graph- and circuit-based approaches provide a means for scaling this up to deliver landscape-scale connectivity statistics. It is important that future work recognizes the strong complementarity of these approaches, and seeks to integrate the strengths of each (Cushman et al. 2013, Kool et al. 2013). One important initial task will be to establish how the landscape statistics provided by graph- and/or circuit theory-based approaches differ depending upon the methods used to provide the internode distances; it will be important to learn for which types of species and for which types of landscapes the difference is substantial, as this will help determine when there will be substantial benefit of investing resources in obtaining the dispersal information required for estimating SMS parameters. 


\section{Perspectives}

Our study showed on two systems that SMS predictions can provide a substantially better model of connectivity than either Euclidean, LCP, or circuit theory-based estimates. One limitation of our study is the rather low number of populations in our systems, and its associated risk of overfitting. It will hence be important to validate our results with independent data on the same species. But also, despite the fact that those two case studies are based on two very different species (a bird and an amphibian), it would be interesting to test the generality of this result by pursuing this comparison and running further tests on more species. It would also be valuable to test the relative performance of SMS in the other type of frequently encountered situation, i.e., when no empirical estimates of cost surfaces are available but a range of alternative cost surfaces are tested (e.g., Wang et al. 2009).

Connectivity modeling is also of crucial importance to predict population spatial dynamics. A further crucial step will be to run spatial population models and compare the outcomes (in terms of, for example, persistence, rate of range expansion, genetic structure) obtained depending upon the model of dispersal assumed (e.g., Euclidean distance, LCP, Circuitscape resistance distance, SMS). Such spatial population models already exist, and SMS could easily be integrated into them (e.g., HexSim; Schumaker 2010). One of those models, RangeShifter, already implements SMS (Bocedi et al. 2014). Further, such simulations can be utilized to determine which of the range of potential metrics provided by methods such as graph and circuit theory are most related to those population-level outcomes on which, as conservation biologists, we typically focus.

\section{ACKNOWLEDGMENTS}

This publication issued from the project TenLamas funded by the French Ministère de l'Energie, de l'Ecologie, du Développement Durable et de la Mer through the EU FP6 BiodivERsA Eranet; by the Agence Nationale de la Recherche (ANR) through the open call INDHET and 6th extinction MOBIGEN to V. M. Stevens, M. Baguette, and A. Coulon, and young researcher GEMS (ANR-13-JSV7-0010-01) to V. M. Stevens and M. Baguette; and by a VLIR-VLADOC scholarship awarded to J. Aben. L. Lens, J. Aben, D. Strubbe, and E. Matthysen are grateful to the Research Foundation Flanders (FWO) for financial support of fieldwork and genetic analysis (grant G.0308.13). V. M. Stevens and M. Baguette are members of the "Laboratoire d'Excellence" (LABEX) entitled TULIP (ANR-10-LABX-41). J. M. J. Travis and S. C. F. Palmer also acknowledge the support of NERC. A. Coulon and J. Aben contributed equally to the work.

\section{Literature Cited}

Aben, J., F. Adriaensen, K. W. Thijs, P. Pellikka, M. Siljander, L. Lens, and E. Matthysen. 2012. Effects of matrix composition and configuration on forest bird movements in a fragmented Afromontane biodiversity hotspot. Animal Conservation 15:658-668.

Aben, J., D. Strubbe, F. Adriaensen, S. C. F. Palmer, J. M. J. Travis, L. Lens, and E. Matthysen. 2014. Simple individualbased models effectively represent Afrotropical forest bird movement in complex landscapes. Journal of Applied Ecology 51:693-702.

Adriaensen, F., J. P. Chardon, G. De Blust, E. Swinnen, S. Villalba, H. Gulinck, and E. Matthysen. 2003. The application of "least-cost" modelling as a functional landscape model. Landscape and Urban Planning 64:233-247.

Baguette, M., S. Blanchet, D. Legrand, V. M. Stevens, and C. Turlure. 2013. Individual dispersal, landscape connectivity and ecological networks. Biological Reviews 88:310-326.

Baguette, M., and H. Van Dyck. 2007. Landscape connectivity and animal behavior: functional grain as a key determinant for dispersal. Landscape Ecology 22:1117-1129.

Bartoń, K. A., T. Hovestadt, B. L. Phillips, and J. M. J. Travis. 2012. Risky movement increases the rate of range expansion. Proceedings of the Royal Society B 279:1194-1202.

Beaumont, M. A. 2010. Approximate Bayesian computation in evolution and ecology. Annual Review of Ecology, Evolution, and Systematics 41:379-406.

Beebee, T. J. C. 1983. The natterjack toad. Oxford University Press, Oxford, UK.

Beerli, P., and J. Felsenstein. 1999. Maximum-likelihood estimation of migration rates and effective population numbers in two populations using a coalescent approach. Genetics 152:763-773.

Beerli, P., and J. Felsenstein. 2001. Maximum likelihood estimation of a migration matrix and effective population sizes in $n$ subpopulations by using a coalescent approach. Proceedings of the National Academy of Sciences USA 98: 4563-4568.

Bélisle, M. 2005. Measuring landscape connectivity: the challenge of behavioral landscape ecology. Ecology 86: 1988-1995.

Bocedi, G., S. C. F. Palmer, G. Pe'er, R. K. Heikkinen, Y. G. Matsinos, K. Watts, and J. M. J. Travis. 2014. RangeShifter: a platform for modelling spatial eco-evolutionary dynamics and species' responses to environmental changes. Methods in Ecology and Evolution 5:388-396.

Callens, T., P. Galbusera, E. Matthysen, E. Y. Durand, M. Githiru, J. R. Huyghe, and L. Lens. 2011. Genetic signature of population fragmentation varies with mobility in seven bird species of a fragmented Kenyan cloud forest. Molecular Ecology 20:1829-1844.

Coulon, A., J. F. Cosson, J. M. Angibault, B. Cargnelutti, M. Galan, N. Morellet, E. Petit, S. Aulagnier, and A. J. M. Hewison. 2004. Landscape connectivity influences gene flow in a roe deer population inhabiting a fragmented landscape: an individual-based approach. Molecular Ecology 13:28412850.

Cushman, S. A., B. McRae, F. Adriaensen, P. Beier, M. Shirley, and K. Zeller. 2013. Biological corridors and connectivity. Pages 384-404 in Key Topics in Conservation Biology 2. John Wiley and Sons, New York, New York, USA.

del Mar Delgado, M., V. Penteriani, V. Nams, and L. Campioni. 2009a. Changes of movement patterns from early dispersal to settlement. Behavioral Ecology and Sociobiology 64:35-43.

del Mar Delgado, M., V. Penteriani, V. O. Nams, and L. Campioni. 2009b. Changes of movement patterns from early dispersal to settlement. Behavioral Ecology and Sociobiology 64:35-43.

Donald, P. F., and A. D. Evans. 2006. Habitat connectivity and matrix restoration: the wider implications of agri-environment schemes. Journal of Applied Ecology 43:209-218.

Fahrig, L. 2003. Effects of habitat fragmentation on biodiversity. Annual Review of Ecology, Evolution, and Systematics 34:487-515.

Glen, A. S., R. P. Pech, and A. E. Byrom. 2013. Connectivity and invasive species management: towards an integrated landscape approach. Biological Invasions 15:2127-2138.

Grimm, V., E. Revilla, U. Berger, F. Jeltsch, W. M. Mooij, S. F. Railsback, H.-H. Thulke, J. Weiner, T. Wiegand, and D. L. DeAngelis. 2005. Pattern-oriented modeling of agent- 
based complex systems: lessons from ecology. Science 310 : 987-991.

Hannah, L. E. E. 2011. Climate change, connectivity, and conservation success. Conservation Biology 25:1139-1142.

Hanski, I. 1998. Metapopulation dynamics. Nature 396:41-49.

Hanski, I., and M. Gilpin. 1991. Metapopulation dynamics: brief history and conceptual domain. Biological Journal of the Linnean Society 42:3-16.

Hodgson, J. A., C. D. Thomas, C. Dytham, J. M. J. Travis, and S. J. Cornell. 2012. The speed of range shifts in fragmented landscapes. PLoS ONE 7:e47141.

Hovestadt, T., B. Binzenhoefer, P. Nowicki, and J. Settele. 2011. Do all inter-patch movements represent dispersal? A mixed kernel study of butterfly mobility in fragmented landscapes. Journal of Animal Ecology 80:1070-1077.

Jeltsch, F., et al. 2013. How can we bring together empiricists and modellers in functional biodiversity research? Basic and Applied Ecology 14:93-101.

Keith, S., E. K. Urban, and C. H. Fry. 1992. Birds of Africa. Volume 4: Broadbills to Chats. Academic Press, London, UK.

Keyghobadi, N. 2007. The genetic implications of habitat fragmentation for animals. Canadian Journal of Zoology 85: 1049-1064.

Kindlmann, P., and F. Burel. 2008. Connectivity measures: a review. Landscape Ecology 23:879-890.

Kool, J., A. Moilanen, and E. Treml. 2013. Population connectivity: recent advances and new perspectives. Landscape Ecology 28:165-185.

Mantel, N. 1967. Detection of disease clustering and a generalized regression approach. Cancer Research 27:209-220.

McRae, B. H., and P. Beier. 2007. Circuit theory predicts gene flow in plant and animal populations. Proceedings of the National Academy of Sciences USA 104:19885-19890.

McRae, B. H., B. G. Dickson, T. H. Keitt, and V. B. Shah. 2008. Using circuit theory to model connectivity in ecology, evolution, and conservation. Ecology 89:2712-2724.

McRae, B. H., V. B. Shah, and T. K. Mohapatra. 2013. Circuitscape 4 user guide. The Nature Conservancy. http:// www.circuitscape.org

Moilanen, A., and I. Hanski. 2001. On the use of connectivity measures in spatial ecology. Oikos 95:147-151.

Moore, J. A., D. A. Tallmon, J. Nielsen, and S. Pyare. 2011. Effects of the landscape on boreal toad gene flow: Does the pattern-process relationship hold true across distinct landscapes at the northern range margin? Molecular Ecology 20: $4858-4869$.

Nabe-Nielsen, J., J. Tougaard, J. Teilmann, K. Lucke, and M. C. Forchhammer. 2013. How a simple adaptive foraging strategy can lead to emergent home ranges and increased food intake. Oikos 122:1307-1316.

Opdam, P., and D. Wascher. 2004. Climate change meets habitat fragmentation: linking landscape and biogeographical scale levels in research and conservation. Biological Conservation 117:285-297.

Palmer, S. C. F., A. Coulon, and J. M. J. Travis. 2011. Introducing a 'stochastic movement simulator' for estimating habitat connectivity. Methods in Ecology and Evolution 2: 258-268.

Pellikka, P. K. E., M. Lotjonen, M. Sijander, and L. Lens. 2009. Airborne remote sensing of spatiotemporal change (1955-2004) in indigenous and exotic forest cover in the Taita Hills, Kenya. International Journal of Applied Earth Observation and Geoinformation 11:221-232.
Revilla, E., T. Wiegand, F. Palomares, P. Ferreras, and M. Delibes. 2004. Effects of matrix heterogeneity on animal dispersal: from individual behavior to metapopulation-level parameters. American Naturalist 164:E130-E153.

SAS. 2010. SAS Version 9.3. SAS Institute, Cary, North Carolina, USA

Schumaker, N. H. 2010. HexSim (Version 1.5.1.14). Environmental Research Laboratory, U.S. Environmental Protection Agency, Corvallis, Oregon, USA.

Schwartz, M. K., J. P. Copeland, N. J. Anderson, J. R. Squires, R. M. Inman, K. S. McKelvey, K. L. Pilgrim, L. P. Waits, and S. A. Cushman. 2009. Wolverine gene flow across a narrow climatic niche. Ecology 90:3222-3232.

Singh, N. J., and G. Ericsson. 2014. Changing motivations during migration: linking movement speed to reproductive status in a migratory large mammal. Biology Letters 10 . http://dx.doi.org/10.1098/rsbl.2014.0379

Spanhove, T. 2012. Avian persistence in a severely fragmented Afrotropical cloud forest. Ghent University, Ghent, Belgium.

Stevens, V. M., E. Leboulengé, R. A. Wesselingh, and M. Baguette. 2006a. Quantifying functional connectivity: experimental assessment of boundary permeability for the natterjack toad (Bufo calamita). Oecologia 150:161-171.

Stevens, V. M., C. Verkenne, S. Vandewoestijne, R. A. Wesselingh, and M. Baguette. 2006b. Gene flow and functional connectivity in the natterjack toad. Molecular Ecology 15:2333-2344.

Taylor, P. D., L. Fahrig, K. Henein, and G. Merriam. 1993. Connectivity is a vital element of landscape structure. Oikos 68:571-573.

Tesson, S., and P. Edelaar. 2013. Dispersal in a changing world: opportunities, insights and challenges. Movement Ecology 1: 10.

Tischendorf, L., and L. Fahrig. 2000. On the usage and measurement of landscape connectivity. Oikos 90:7-19.

Travis, J. M. J., et al. 2013. Dispersal and species' responses to climate change. Oikos 122:1532-1540.

Urban, D. L., E. S. Minor, E. A. Treml, and R. S. Schick. 2009. Graph models of habitat mosaics. Ecology Letters 12:260 273.

Van Dyck, H., and M. Baguette. 2005. Dispersal behaviour in fragmented landscapes: routine or special movements? Basic and Applied Ecology 6:535-545.

Wang, I. J., W. K. Savage, and H. B. Shaffer. 2009. Landscape genetics and least-cost path analysis reveal unexpected dispersal routes in the California tiger salamander (Ambystoma californiense). Molecular Ecology 18:1365-1374.

Wang, J. L., and M. C. Whitlock. 2003. Estimating effective population size and migration rates from genetic samples over space and time. Genetics 163:429-446.

Wiegand, T., F. Jeltsch, I. Hanski, and V. Grimm. 2003. Using pattern-oriented modeling for revealing hidden information: a key for reconciling ecological theory and application. Oikos 100:209-222.

Wiens, J. A. 2001. The landscape context of dispersal. Pages 96-99 in J. Clobert, E. Danchin, A. A. Dhondt, and J. D. Nichols, editors. Dispersal. Oxford University Press, New York, New York, USA.

Zeller, K., K. McGarigal, and A. Whiteley. 2012. Estimating landscape resistance to movement: a review. Landscape Ecology 27:777-797.

Zollner, P. A., and S. L. Lima. 1999. Search strategies for landscape-level interpatch movements. Ecology 80:10191030.

\section{Supplemental Material}

\section{Ecological Archives}

Appendices A-H and Supplements 1-3 are available online: http://dx.doi.org/10.1890/14-1690.1.sm 